\title{
CORRIGENDUM
}

\section{Specificity and mechanism-of-action of the JAK2 tyrosine kinase inhibitors ruxolitinib and SAR302503 (TG101348)}

T Zhou, S Georgeon, R Moser, DJ Moore, A Caflisch and O Hantschel

Leukemia (2014) 28, 471-472; doi:10.1038/leu.2013.299

Correction to: Leukemia (2014) 28, 445-448; doi:10.1038/ leu.2013.205; published online 19 July 2013

In the original publication of the article, the authors have inadvertently used a structural variant of the JAK2 kinase inhibitor

a

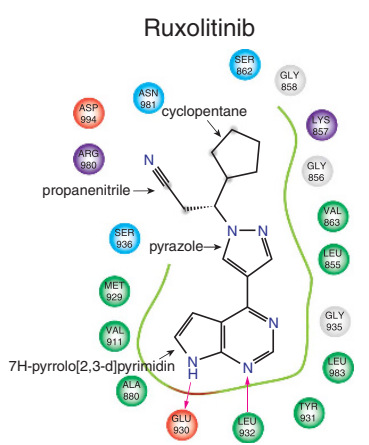

b

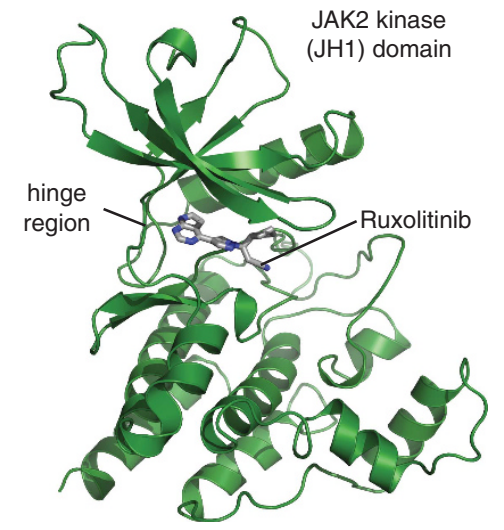

C

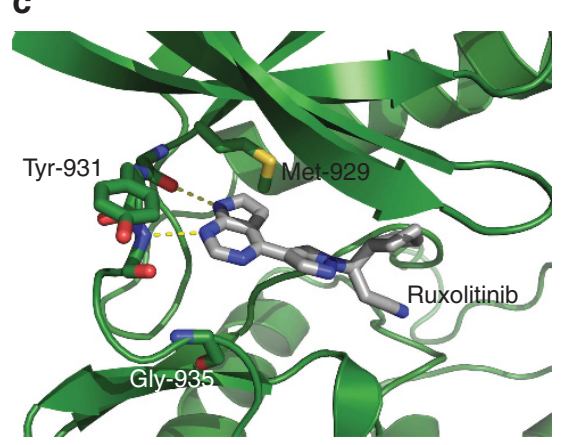

SAR302503 (TG101348) for the molecular dynamics simulations shown in Figure 1 (right panels). This structural variant is published on some webpages of chemical vendors and on www.wikipedia.org, but is not identical to SAR302503, as it contains an amino group instead of a methyl group as a

SAR302503
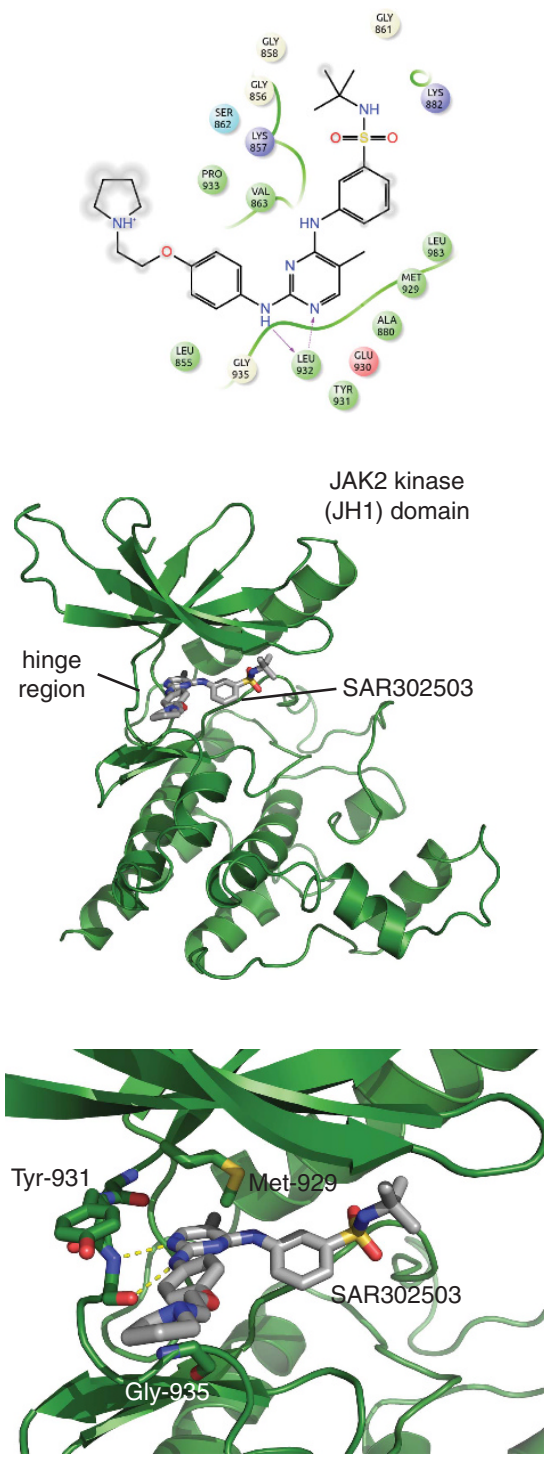

Figure 1. For legend please see page 472. 
substituent on the pyrimidine ring of SAR302503 and two of its rings are linked with a methylene instead of a amine linkage.

The authors have repeated molecular dynamics simulations with the correct structure of SAR302503 and predict a binding mode that is very similar to the proposed binding mode and of the recently published crystal structure of the SAR302503 precursor compound TG101209 in complex with the JAK2 kinase domain (PDB entry 4JI9).

Importantly, the specificity testing of SAR302503 was done with the correct compound and none of the conclusions of our publication are affected by this unfortunate mistake.

The corrected Figure is shown here.

The errors have now been rectified, and the correct article appears in this issue. The html and online pdf versions have also been rectified, and now carry the correct paper. This also includes minor changes within the text itself, and the supplementary information file, which reflect the above amendments.

The authors apologize for any inconvenience caused.

Figure 1. The predicted binding mode of ruxolitinib (left) and SAR302503 (right) to the JAK2 kinase domain. (a) Most populated binding mode of ruxolitinib (left) and SAR302503 (right) in the MD simulations. These two-dimensional plots were prepared with Ligplot. ${ }^{17}$ They show the JAK2 residues in van der Waals contact with the drugs (green, cyan, blue, red and white circles for hydrophobic, polar, basic, acidic and glycine residues, respectively), the intermolecular hydrogen bonds (magenta arrows) and the atoms of the drugs exposed to solvent (gray circles). Ring systems and functional groups of ruxolitinib that are mentioned in the text are labeled. (b) Cartoon representation of the JAK2 kinase domain bound to ruxolitinib (left) and SAR302503 (right). As in panel a, the most populated binding mode of the drugs in the MD simulations is shown. (c) Detailed view of the drug-binding sites. Critical hydrogen bonds to the hinge region are indicated by a yellow dotted line. Met-929 (gatekeeper residue) as well as Tyr-931 and Gly-935 that were shown to render JAK2 resistant to ruxolitinib and SAR302503 upon mutation, are shown as sticks. A detailed description of the employed methods and results can be found in Supplementary Data. 